The Journal of

Thoracic and Cardiovascular Surgery

Vol 123, No. 6, June 2002

\title{
Where are we going with computer-assisted or robotic cardiac surgery? A piece of the totally endoscopic coronary bypass puzzle
}

Randall K. Wolf, MD

See related article on page 1125 .

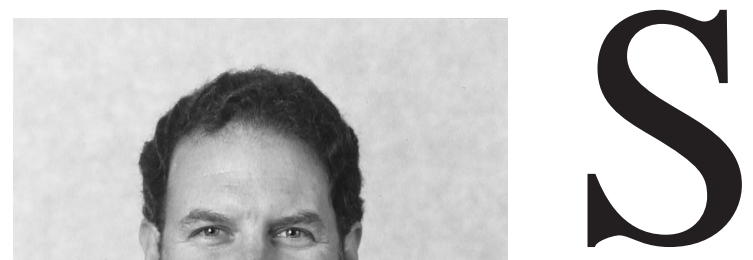

I don't skate to where the puck is; I skate to where the puck is going.

-Wayne Gretzky

The proposed advantage to e-CABG was no different from the advantage of general thoracoscopy over open techniques-to decrease the trauma of the operation and therefore decrease the morbidity related to conventional transthoracic access of the heart and aorta. It was postulated that endoscopic beating heart and left internal thoracic artery (LITA) to left anterior descending (LAD) coronary artery bypass could provide a durable, truly minimally invasive bypass procedure that would likely be superior in the long term to current interventional approaches to the diseased LAD.

Experimentation and clinical experience with modifications of general thorascopic techniques proved that the LITA could routinely be mobilized thoracoscopically, but that standard thorascopic instruments were inadequate for any usual or customary anastomotic technique. ${ }^{1-3}$ It became clear that the endoscopically performed anastomosis was a great challenge in completing e-CABG.

The solution to this endoscopic anastomotic dilemma centered around three new (to cardiac surgeons) technologies: robotics, automatic distal anastomotic devices, and sealants. The first anastomotic technology to be tested clinically to any great extent has been robotics, which is really computer-assisted technology. The robot is not autonomous. The essence of this robotic technology in cardiac surgery is that for the first time the surgeon's hand motions are captured and transformed into a binary code, allowing computers to modify the signals and transmit the signals to tiny instruments inside the chest cavity. This is triumph of technology in the operating room.

The fine motor control of the surgeon's hand is transmitted to tiny instruments that can now facilitate an endoscopic anastomosis. The control is so perfect that cardiac surgeons are able to construct an anastomosis in the customary fashion without placing a hand inside the chest cavity. Indeed, the surgeon is not scrubbed, but sits comfortably at a console, peering into the chest with 3-dimensional vision.

The work of Dogan and associates ${ }^{4}$ is important for two reasons. Their study 
demonstrates that e-CABG can be performed in certain clinical settings. As my friend John Flege has pointed out, this is proof of concept (personal communication, 2001). The Wright brothers did not prove one could fly coast-tocoast in a Boeing 747 . They proved with technology that human beings could fly. Refinements in and evolution of aviation technology have occurred over many years. Second, as the current article concludes, there is need for much improvement. Nevertheless, the concept of computer-assisted or robotic CABG has been proven.

This technique is the sentinel event that heralds enabling virtual reality technology in the cardiac operating suite. The surgeon visualizes the heart indirectly through a 3-dimensional video screen bringing cardiac surgery into the 21 st century. Although this technology holds great promise, there are still major technological hurdles. Some of these hurdles may be overcome by one of the other techniques mentioned above, namely, automatic distal anastomotic devices or quick connectors. Initial clinical trials with a few of these connectors have begun in open CABG cases, but the devices certainly will be tested in the minimally invasive arena next. Initial experience reveals they can be deployed with about 30 seconds of coronary occlusion time and consistently result in a widely patent anastomosis.

Sealants may also aid in the distal anastomosis, but their role at this juncture is unclear. With quick connect devices and minimal access technology such as robotics and endoscopic stabilizers, more coronary target sites (besides the LAD) may be accessed routinely. However, even if only the LAD can be accessed endoscopically on the beating heart in a routine fashion, this would be a huge step in decreasing the trauma of surgical coronary revascularization and could compete as a best solution for selected patients with obstructive coronary artery disease. This would include hybrid procedures, in which the LITA is mobilized endoscopically and anastomosed to the LAD, and other lesions are addressed by catheter-based techniques.

In the near future, I predict that the LITA will be harvested endoscopically by a growing number of cardiac surgeons. In the past, it was difficult to teach endoscopic ITA mobilization to cardiac surgeons who generally had little or no endoscopic training. With computer-assisted systems, teaching cardiac surgeons endoscopic ITA mobilization is much easier. The anastomosis between the LITA and LAD then might be performed with a quick connect device, either through a small-access thoracotomy or completely endoscopically. Endoscopic stabilizers have evolved significantly and adequate stabilization is now routine. In addition, multiple consoles with additional manipulator arms will allow two surgeons to operate together in a robotic scenario. ${ }^{5}$ In the long term, perhaps multiple bypasses will be performed on the beating heart.

It is appropriate and fitting to place a word of caution against widespread use of robotics in heart surgery without further careful clinical studies such as the current article by Dogan and associates. The concerns of generations of surgeons trained in open techniques should not be underestimated. They are valid and need to be addressed in an unbiased manner. Surgeons should continue to be optimistic and excited about the future of computer-assisted technology in cardiac operations, realizing that they may play a role in solving the puzzle of e-CABG. With further refinement in quick connect devices and stabilizers, our specialty may be able to ensure careful evolution and proper clinical use in this new technology. I predict that, as a result of research in e-CABG technologies, the role of conventional suturing in performing coronary anastomoses will decrease and the use of automatic anastomotic devices or quick connectors will increase. Wayne Gretzky also said, "You miss $100 \%$ of the shots you don't take." It is refreshing to witness cardiac surgeons taking a few more shots at this evolving technology. We are striving toward less traumatic cardiac procedures. Ultimately our patients will benefit from this evolution of technology and technique.

\section{References}

1. Ohtsuka T, Wolf RK, Hiratzka LF, Wurnig P, Flege JB. Thoracoscopic internal mammary artery harvest for MICABG using the Harmonic Scalpel. Ann Thorac Surg. 1997;63:S107-9.

2. Wolf RK, Ohtsuka T, Flege JB. Early results of thoracoscopic internal mammary artery harvest using an ultrasonic scalpel. Eur J Cardiothorac Surg. 1998;14:S54-7.

3. Duhaylongsod FG, Mayfield WR, Wolf RK. Thoracoscopic harvest of the internal thoracic artery: a multicenter experience with 218 cases. Ann Thorac Surg. 1998;66:1012-7.

4. Dogan S, Aybek T, Andreßen E, Byhahn C, Mierdl S, Westphal K, et al. Totally endoscopic coronary artery bypass grafting on cardiopulmonary bypass with robotically enhanced telemanipulation: report of forty-five cases. J Thorac Cardiovasc Surg. 2002;123:1125-31.

5. Falk V, Fann JI, Grunenfelder J, Daunt D, Burdon TA. Endoscopic computer-enhanced beating heart coronary artery bypass grafting. Ann Thorac Surg. 2000;70:2029-33. 